\title{
Extracting heartrate from optical signal of functional near-infrared spectroscopy based on mathematical morphology
}

\author{
Jinyan Sun ${ }^{* \dagger}, \boldsymbol{\oplus}$, Linshang Rao* and Chenyang $\mathrm{Gao}^{\S}$ \\ *Department of Biomedical Engineering \\ Guangdong Medical University, Dongguan 523808, China \\ ${ }^{\dagger}$ School of Stomatology and Medicine \\ Foshan University, Foshan 528000, China \\ ${ }^{*}$ School of Biomedical Engineering \\ Southern Medical University, Guangzhou 510515, China \\ ${ }^{\S}$ Britton Chance Center for Biomedical Photonics \\ Wuhan National Laboratory for Optoelectronics-Huazhong \\ University of Science and Technology, Wuhan 430074, China \\ \bmesunjinyan@sina.cn
}

Received 2 September 2017

Accepted 19 November 2017

Published 2 January 2018

\begin{abstract}
Functional near-infrared spectroscopy (fNIRS), as a new optical functional neuroimaging method, has been widely used in neuroscience research. In some research fields with NIRS, heartrate (HR) (or heartbeat) is needed as useful information to evaluate its influence, or to know the state of subject, or to remove its artifact. If HR (or heartbeat) can be detected with high accuracy from the optical intensity, this will undoubtedly benefit a lot to many NIRS studies. Previous studies have used the moving time window method or mathematical morphology method (MMM) to detect heartbeats in the optical intensity. However, there are some disadvantages in these methods. In this study, we proposed a method combining the periodic information of heartbeats and the operator of mathematical morphology to automatically detect heartbeats in the optical intensity. First the optical intensity is smoothed using a moving average filter. Then, the opening operator of mathematical morphology extracts peaks in the smoothed optical intensity. Finally, one peak is identified as a heartbeat peak if this peak is the maximum in a predefined point range. Through validation on experimental data, our method can overcome the disadvantages of previous methods, and detect heartbeats in the optical signal of fNIRS with nearly $100 \%$ accuracy.
\end{abstract}

Keywords: Functional near-infrared spectroscopy; heartrate; mathematical morphology.

『Corresponding author.

This is an Open Access article published by World Scientific Publishing Company. It is distributed under the terms of the Creative Commons Attribution 4.0 (CC-BY) License. Further distribution of this work is permitted, provided the original work is properly cited. 


\section{Introduction}

Functional near-infrared spectroscopy (fNIRS) is a noninvasive optical functional neuroimaging method, and can measure the concentration changes of oxy-hemoglobin $(\Delta[\mathrm{HbO} 2])$ and deoxy-hemoglobin $(\Delta[\mathrm{Hb}])$ to reflect relative regional brain activity. Along with the development of methods for processing fNIRS data, ${ }^{1-3}$ fNIRS has been wildly used in cognitive neuroscience, ${ }^{4-6}$ disease, ${ }^{7}$ infant $^{8}$ and hyperscanning studies ${ }^{9}$ due to its flexibility and high ecological validity, and obtained significant results. In studies using $\Delta[\mathrm{HbO} 2]$ and $\Delta[\mathrm{Hb}]$ to represent the brain activity, heartbeat is the most dominant physiological noise, and should be filtered or removed directly. 5,10

There are also many other fNIRS studies using heartrate (HR) as useful information. For example, some studies investigated the influence of physiological signals (such as HR) on fNIRS measurement. ${ }^{11-13}$ Some other fNIRS studies need HR information to know the state of subjects. For example, Durantin et al. used NIRS and HR variability (HRV) to study the mental overload, and their results showed that both NIRS and HRV were sensitive to different levels of mental workload. ${ }^{14}$ Tanida et al. used NIRS to study the mental stress, and they recorded HR simultaneously to verify the elicitation of stress. ${ }^{15}$ Nowadays, most of these studies recorded HR with supplementary instruments. This increased workload and cost of researches.

Some NIRS studies also need heartbeat information to remove its artifacts, like studies on fast neuronal signal. Nowadays, many researchers try to measure the fast neuronal signal with NIRS noninvasively. ${ }^{16-18}$ The fast neuronal signal can localize the brain activity with a high temporal resolution $(<100 \mathrm{~ms})$ and directly reflect the functional activity of nerves, ${ }^{18}$ which opens a lot of new possibilities for neuroscience research. To obtain the fast neuronal signal from the optical intensity, the first important step is removing the artifacts produced by heartbeat. To do this, some studies calculated the average heartbeat by detecting each heartbeat, adapted its length to each heartbeat and subtracted it from each individual heartbeat. This method can effectively remove the influence of heartbeats. ${ }^{16,19}$

Researchers have developed two methods to obtain the average heartbeat in NIRS studies on fast neuronal signal. One method used the moving time window method (MTWM) to identify each heartbeat by finding the minimum of the smoothed time derivative of the optical signal within each time window. ${ }^{19}$ Although this method is easy to carry out, a proper window length (WL) is difficult to set. Besides, a fixed length of the time window is difficult to adapt to changes in HR, and induces influence in detecting adjacent heartbeats. Recent studies have used the mathematical morphology method (MMM) to help find each heartbeat peak. ${ }^{16}$ By setting a threshold, one peak with amplitudes larger than the threshold was marked as heartbeat peak. This method can work well for many optical data. But, a threshold could be chosen improperly due to the influence of extreme values for some optical data, and an improper threshold causes increase in heartbeat miss. Thus, it is necessary to develop a new method to accurately detect each heartbeat in the optical intensity.

Taken together, in many research fields with NIRS, HR (or heartbeat) is needed as useful information to evaluate its influence, or to know the state of subject, or to remove its artifact. If HR (or heartbeat) can be detected with high accuracy from the optical intensity of NIRS, it will save workload and cost of many researches. In the same time, the subjects might feel more comfortable without more extra instruments. From this perspective, detecting HR (or heartbeat) from optical intensity of NIRS will undoubtedly benefit a lot to many studies. In this study, we developed a method combining the periodic information of heartbeats and the opening operator of mathematical morphology to automatically extract HR in the optical intensity of NIRS.

\section{Method}

The data used to test our method was the optical intensity of NIRS in a color-word matching Stroop task from our published study. ${ }^{5}$ The Stroop task lasted about $28 \mathrm{~min}$. The optical intensity were acquired with a continuous-wave fNIRS system ${ }^{20}$ developed by the Britton Chance Center for Biomedical Photonics, and sampled at $70 \mathrm{~Hz}$.

Our method has the following five steps to extract the HR.

\section{(1) Smooth optical intensity}

The raw optical intensity (represented by variable data) was first smoothed using a 5-point moving average filter (data_s) to reduce the influence of noise. 
(2) Extract peaks using opening operator of mathematical morphology

For opening operator of mathematical morphology, a linear structuring element $g$ is used. The amplitude of $g$ is one, and its length $(M)$ is $f_{s} / f_{\text {heart }}$, where $f_{s}$ is the sampling frequency and $f_{\text {heart }}$ is the mean HR of the subject. For 1D signal data_s, the definition of erosion operator and dilation operator are expressed by Eqs. (1) and (2). Compute the opening (Eq. (3)) of data_s,${ }^{21,22}$ that is dilation operator (Eq. (2)) after erosion operator (Eq. (1)), and subtract the opening of data_s from data_s, to get an output (data_s_m) consisting of all optical signal peaks.

$$
\begin{gathered}
\left(\text { data } \_s \Theta g\right)(n)=\min \{\text { data_s }(n+m)-g(m)\}, \\
(\text { data_s } \oplus g)(n)=\max \{\text { data_s }(n-m)+g(m)\},
\end{gathered}
$$

where $m \in 0,1,2, \ldots, M-1, n+m, n-m \in 0$, $1,2, \cdots, N-1, N$ is the length of data_s.

$$
\left(\text { data } \_s \circ g\right)(n)=\left(\text { data } \_s \Theta g \oplus g\right)(n) .
$$

(3) Find all peaks in data_s_m

Find all local peaks in data_s_ $m$ by using the findpeaks function in Matlab.

(4) Determine whether a peak is a heartbeat peak One peak in data_s_ $m$ is identified as a heartbeat peak if this peak value is the maximum in a point range of $\left[p e a k_{-} l o c-r\right.$, peak_loc $\left.+r\right]$, where peak_loc is the point location of the peak value, $r$ is $0.5^{*} f_{s} /$ $f_{\text {heart_Nmin }}$ and is rounded to the nearest integer. $f_{\text {heart_Nmin }}$ is the mean HR of the $N$ th minute of the peak time. For example, if one peak value is located between 1 and $2 \mathrm{~min}, f_{\text {heart_Nmin }}$ is the mean HR of data between $1 \mathrm{~min}$ and $2 \mathrm{~min}$. So $r$ is kept updated according to the time where each peak is. The locations of heartbeat peaks in data_s_ $m$ are also the locations of heartbeat peaks in data_s.

(5) Obtain the HR

Obtain the HR information through each heartbeat.

Figure 1 shows the schematic diagram of our method. To evaluate the applicability of our method, we used power spectral density ratio (PSDR, Eq. (4)) to assess the signal quality of optical intensity, and explored the detection results of our method for optical intensity with different signal qualities. Considering the characteristics of our data, the power spectral density (PSD) in the frequency range of $0.5-2.5 \mathrm{~Hz}$

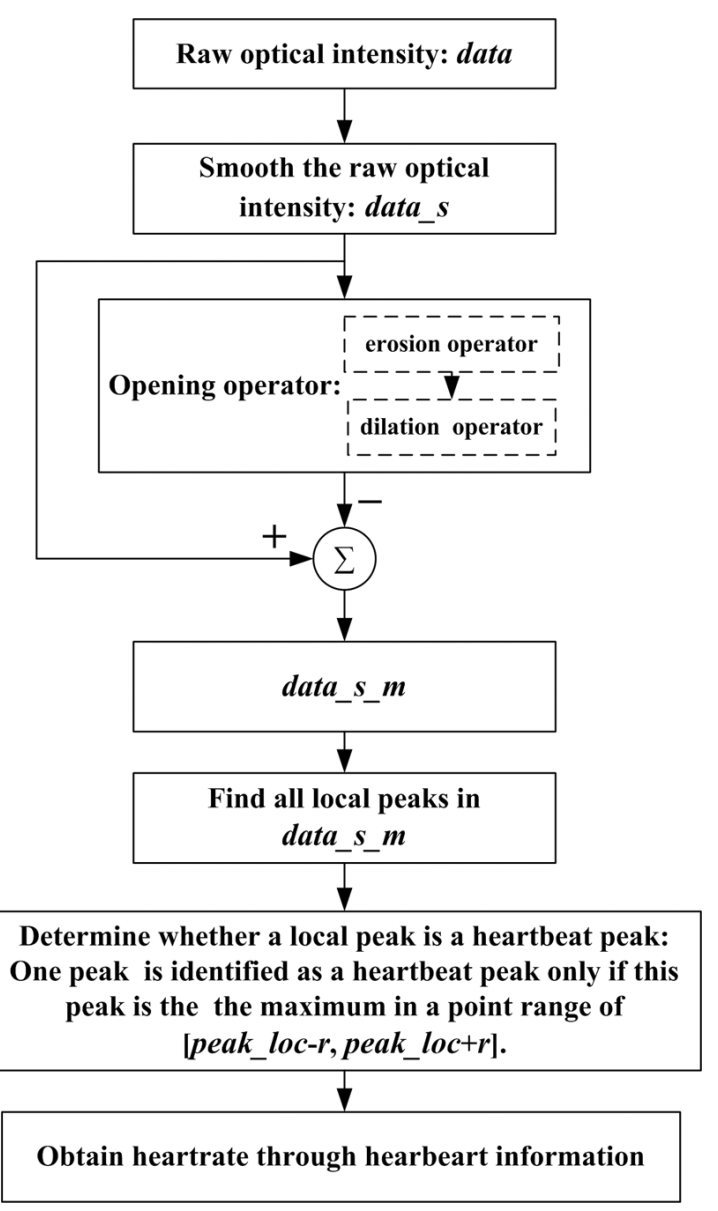

Fig. 1. The schematic diagram of our method for extracting $\mathrm{HR}$ in the optical intensity.

was used to represent the heartbeat PSD. The PSD in the frequency range of $0.5-f_{s} / 2 \mathrm{~Hz}$ was used to represent the total PSD since signal below heartbeat frequency has little influence on heartbeat detection. When PSDR is low, the judgement of each heartbeat is easily influenced by noise. So we only analyzed the detection results for some typical channels with relative high PSDR. The detection results were evaluated by analyzing the heartbeat accuracy and miss rate separately. In addition, we compared our methods with the existing two methods.

$$
\mathrm{PSDR}=\frac{\int_{0.5}^{2.5} \mathrm{PSD}}{\int_{0.5}^{f_{s} / 2} \mathrm{PSD}} .
$$

\section{Results}

Figure 2 shows the original optical intensity with different PSDR over a typical period of time from 

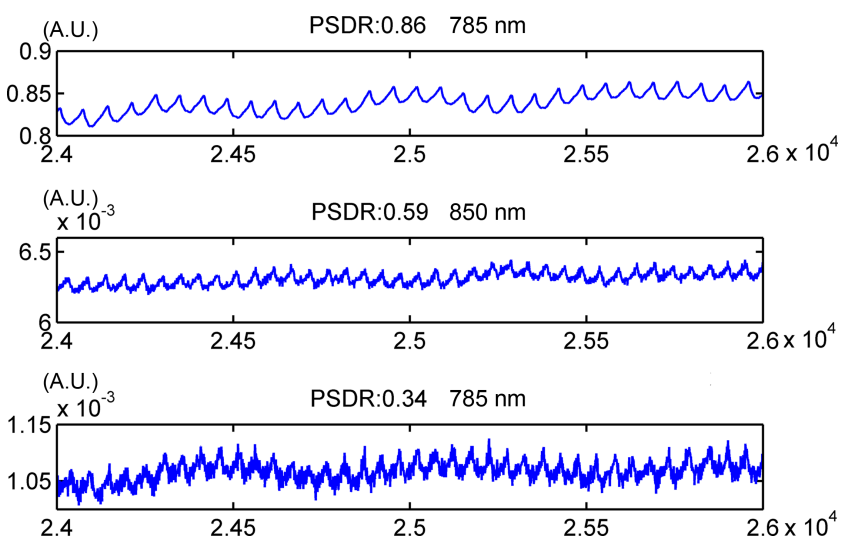

Fig. 2. The original optical intensity over a typical period of time from three subjects with different PSDR. The data in each subgraph is from one channel of one subject. The horizontal axis represents time in points.

three subjects. Figure 2 illustrates that optical intensity with large PSDR has a high signal noise ratio overall, and optical intensity with small PSDR has a low signal noise ratio overall. PSDR can evaluate the signal quality.

Figure 3 illustrates the results after step (1) step (4) of our method. Figure 3(b) shows that all
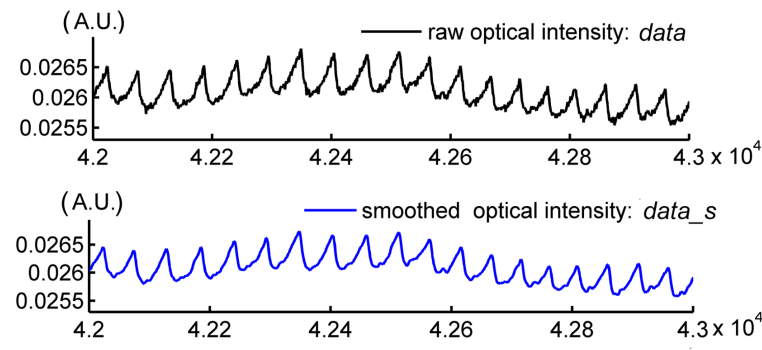

(a)

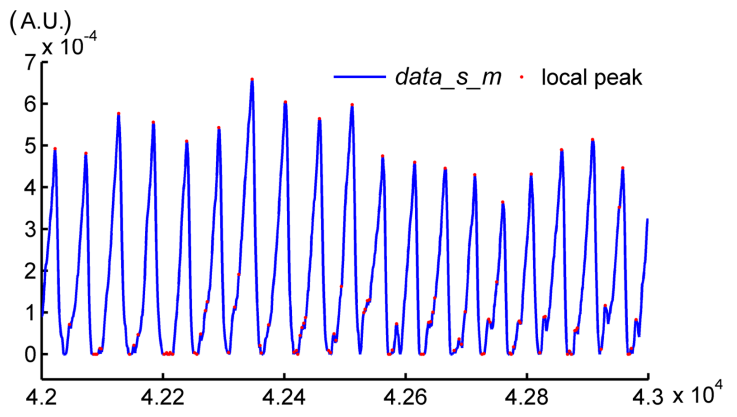

(c) optical signal peaks have been extracted by using the opening operator of mathematical morphology. Figure 3(d) shows that heartbeats have been detected accurately with our method.

In step (4), when $r$ is too small, the error rate would increase, while when $r$ is too large, the miss rate would increase. Through comparative analysis, we set $r$ as $0.5^{*} f_{s} / f_{\text {heart_}} N$ min . The detection results in Table 1 show that heartbeats can be detected with accuracy larger than $99.5 \%$.

Figure 4 shows comparison between our method and the previous MTWM with data from Sub2 Ch4 ${ }^{\mathrm{a}}$. As shown in Figs. 4(a) and 4(b), our method can adapt to the characteristics of data, and accurately detect heartbeat peaks even if $\mathrm{HR}$ varies. To ensure that the heartbeat number detected by MTWM approximates to the actual result, WL is firstly set as 55. As shown in Fig. 4(d), the heartbeat might be missed or repeatedly detected in the same epoch when HR varies for this WL. When WL is smaller, the probability of including two heartbeats in the same epoch increases (Fig. 4(e)). While when WL is larger, the probability of missing a heartbeat increases (Fig. 4(f)).

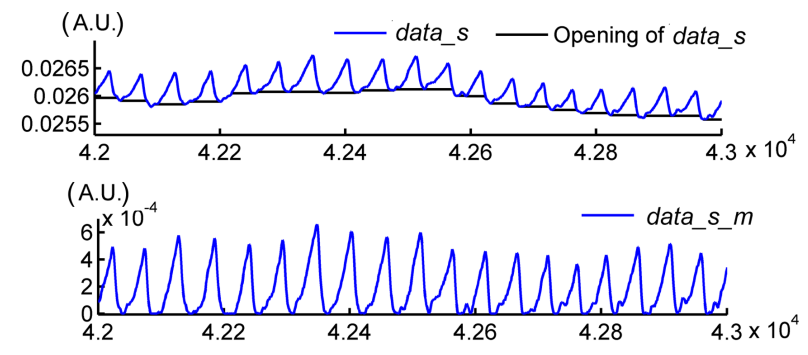

(b)
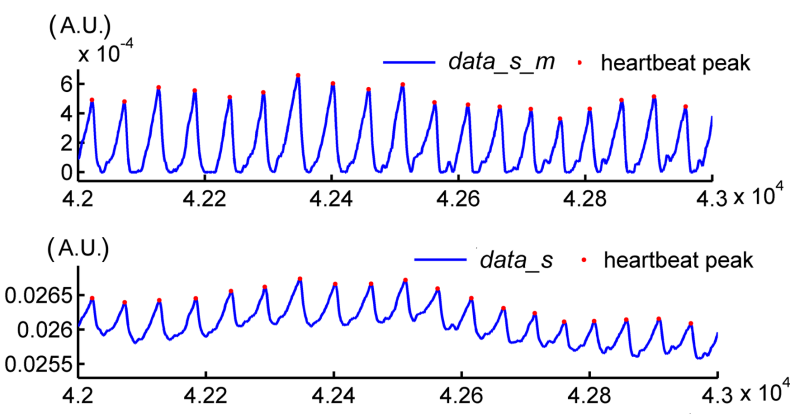

(d)

Fig. 3. Illustration of our method. (a) The raw optical intensity (data) and the smoothed optical intensity (data_s) after step (1). (b) data_s and its opening (top), and the residual signal data_s_m after step (2). (c) All local peaks are found in data_s_m after step (3) and marked with red dots. (d) All heartbeat peaks are identified in data_s_ $m$ after step (4) and marked with red dots in data_s_ $m$ (top) and in data_s (bottom). Figure 3 shows the results over a typical period of time from one channel of one subject. The horizontal axis represents time in points. 


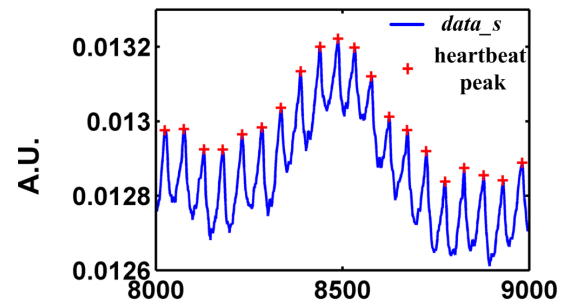

(a)

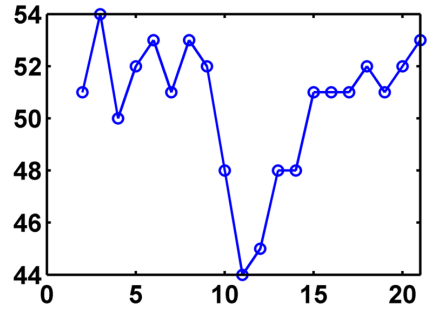

(d)

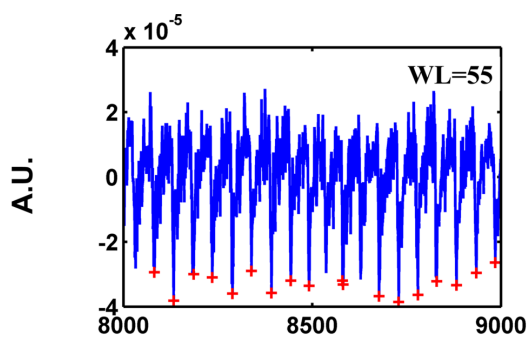

(b)

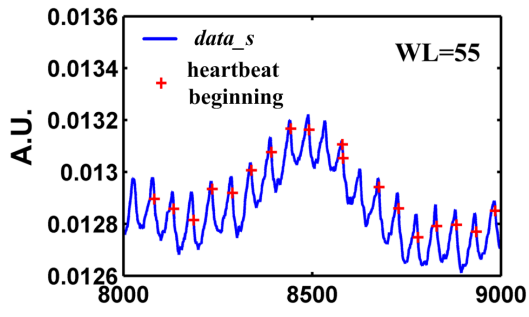

(e)

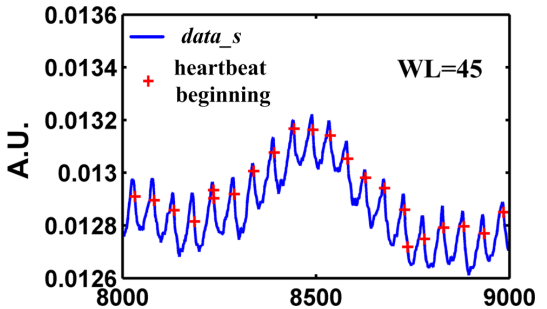

(c)

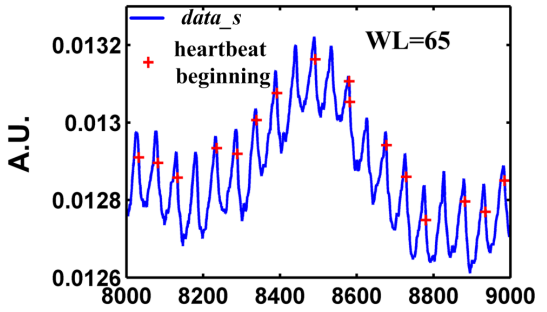

(f)

Fig. 4. Comparison between our method and the MTWM. (a) Heartbeat peaks obtained by our method, and the corresponding heart peak interval is shown in (b). (c) Minimums (red crosses) within a predefined window detected by the MTWM in the smoothed time derivative of optical data. (d) Each minimum is the beginning of each heartbeat, marked in data_s. (e) and (f) show detection results of the MTWM with different WL. The data in (a), (d), (e) and (f) are from Sub2 Ch4 ${ }^{\text {a }}$, and the horizontal axis represents time in points

Figure 5 shows comparison between our method and the previous MMM. Figure 5(a) shows that our method can accurately detect heartbeat peaks from optical intensity with low-frequency drifting and artifacts. The detection accuracy in Fig. 5(a) is
100\%. As shown in Fig. 5(c), the MMM can work well for data when setting a threshold as the average amplitude of the adjacent two peak points generating the largest increment in data_s_m. The detection accuracy in Fig. 5(c) is $100 \%$. But,
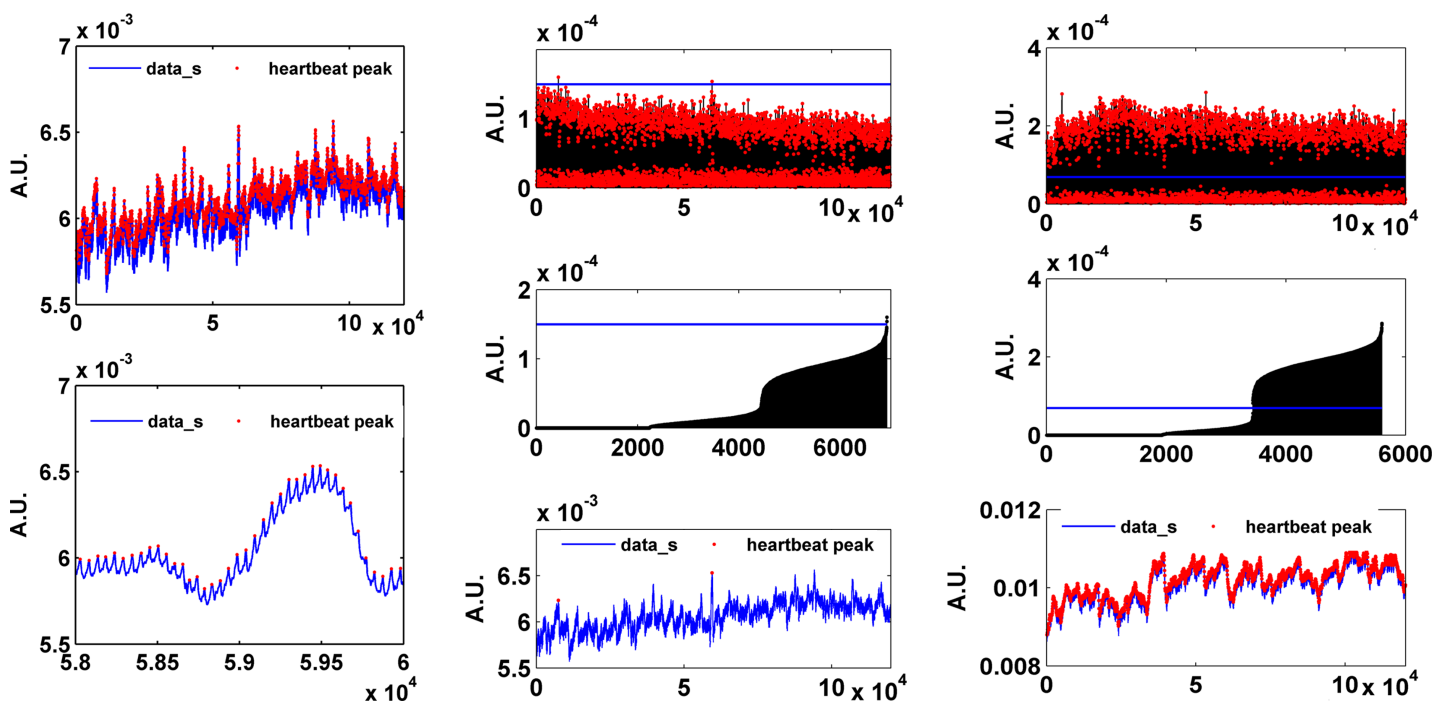

(a)

(b)

(c)

Fig. 5. Comparison between our method and the previous MMM. (a) Heartbeat peaks detected by our method, and the enlarged plot of the corresponding period in the bottom subgraph. (b) Heartbeat peaks obtained by MMM. The top subgraph shows all peaks of waves containing a width smaller $M$ in data_s_ $m$. The middle subgraph shows all peaks in ascending order of amplitude. The blue line in these two subgraphs indicates the threshold. The optical data in (a) and (b) are from Sub3 Ch6 ${ }^{\text {a }}$. (c) Heartbeat peaks obtained by MMM for another data from Sub1 Ch2 ${ }^{\mathrm{b}}$. 
extreme values in data_s_m might cause improper selection of a threshold, and result in missing of heartbeat peaks, as shown in Fig. 5(b).

\section{Discussion}

In this study, we proposed a method to detect each heartbeat to obtain HR in the optical intensity by combining the opening operator of mathematical morphology and the periodic information of heartbeats. Table 1 demonstrates that the detection accuracy of our method is larger than $99.5 \%$ for 28-min data sampled at $70 \mathrm{~Hz}$.

We used the opening operator of mathematical morphology to extract heartbeat peaks, as used in many other fields. ${ }^{22,23}$ The use of opening operator of mathematical morphology can minimize the influence of noise (such as low-frequency drifting and movement artifacts) in detecting heartbeat peaks, as shown in Fig. 5(a). Besides, we used the periodic information of heartbeat, and judged one peak as a heartbeat peak only when it is the maximum over a period of time, which can reduce the influence of the movement artifacts with similar amplitude to heartbeat peaks to a great extent. So extreme values in data_s_m have little influence on heartbeat detection for our method. But extreme values in data_s_ $m$ can cause an improper threshold, and induce increase in heartbeat miss for MMM.

For MTWM, the WL has a significant influence on detection results, and different WL causes different detection number of heartbeats. Besides, the location of a preceding heartbeat has a great influence on subsequent heartbeats. As indicated in Fig. 4, the misjudgment of including two heartbeats in the same epoch rises for a small WL, and the misjudgment of missing a heartbeat rises for a large WL. So it is difficult to choose a proper WL. Even a proper WL cannot avoid the detection error when HR varies. While there is little influence in detecting heartbeat peaks between adjacent heartbeats in our method. In addition, in step (4) of our method, $r$ is relative to the instant $\mathrm{HR}$ of subject, and this reduce the influence of the HR change (variation in HR across the entire experiment for one subject, or difference in HR across subjects) on heartbeat detection. From results in Table 1, it can be drawn that heartbeat can be detected with high accuracy even if there is considerable change in the HR. It should be noted that $f_{\text {heart_Nmin }}$ could be modified according to the specific experimental condition. The instant HR information could be gained after the detection of each heartbeat.

Our method can be used in many NIRS studies that needs HR as useful information. The detection of HR without supplementary instruments can reduce workload and cost of these researches. Subjects might feel more comfortable without more extra instruments, which might be important for some NIRS studies, like studies on emotion or patients. Our method can also be used in NIRS studies on fast neuronal signal. The detection results in Table 1 show that our method can detect heartbeats in the optical signal of fNIRS with accuracy

Table 1. The detection results of our method to data with different PSDR when $r=0.5^{*} f_{s} / f_{\text {heart_Nmin }}$.

\begin{tabular}{|c|c|c|c|c|c|}
\hline Data & Total points (Total heartbeat) & PSDR & Mean HR & Accuracy & Miss rate \\
\hline Sub1 Ch4 ${ }^{\mathrm{a}}$ & $120160(1933)$ & 0.7289 & 67.56 & $99.845 \%$ & $0.155 \%$ \\
\hline Sub1 Ch4 ${ }^{\mathrm{b}}$ & & 0.6210 & 67.56 & $99.845 \%$ & $0.155 \%$ \\
\hline Sub1 Ch12 ${ }^{\mathrm{a}}$ & & 0.8623 & 67.56 & $99.948 \%$ & $0.052 \%$ \\
\hline Sub1 Ch12 ${ }^{\mathrm{b}}$ & & 0.8181 & 67.56 & $99.897 \%$ & $0.103 \%$ \\
\hline $\mathrm{Sub} 2 \mathrm{Ch} 4^{\mathrm{a}}$ & $119530(2153)$ & 0.6704 & 75.65 & $99.954 \%$ & $0.046 \%$ \\
\hline $\mathrm{Sub} 2 \mathrm{Ch} 4^{\mathrm{b}}$ & & 0.4012 & 75.65 & $99.861 \%$ & $0.139 \%$ \\
\hline Sub2 Ch5 ${ }^{\mathrm{a}}$ & & 0.8348 & 75.65 & $100 \%$ & 0 \\
\hline Sub2 $\mathrm{Ch} 5^{\mathrm{b}}$ & & 0.7372 & 75.65 & $100 \%$ & 0 \\
\hline Sub3 Ch2 ${ }^{\mathrm{a}}$ & $119810(2237)$ & 0.5779 & 78.42 & $99.911 \%$ & $0.089 \%$ \\
\hline Sub3 $\mathrm{Ch} 2^{\mathrm{b}}$ & & 0.4697 & 78.42 & $99.821 \%$ & $0.179 \%$ \\
\hline Sub3 Ch4 ${ }^{\mathrm{a}}$ & & 0.7035 & 78.42 & $100 \%$ & 0 \\
\hline Sub3 Ch4 ${ }^{\mathrm{b}}$ & & 0.5904 & 78.42 & $99.955 \%$ & $0.045 \%$ \\
\hline
\end{tabular}

Note: The total points are different between subjects (Sub1, Sub2 and Sub3) due to the slight different recoding time before and after the Stroop task. Ch is short for channel. Superscript a, b means $785 \mathrm{~nm}$ $(850 \mathrm{~nm})$ for the source wavelength in one channel. Mean HR is the average HR (heartbeats/min) across the entire experiment. 
nearly $100 \%$ along with very low miss rate. These results demonstrate that our method can be effectively used in data preprocessing to detect fast neuronal signal by NIRS.

In conclusion, our method can overcome the disadvantages of previous methods, and accurately detect each heartbeat in the optical intensity. This method can gain the subject's HR, and help to know the state of subjects without additional devices. In the future, we are going to compare the detection results by our method with those measured by commercial pulse oximeter to further verify the validity and correctness of our method.

\section{Conflict of Interest}

We declare that there are no conflicts of interest related to this paper.

\section{Acknowledgment}

The authors would like to acknowledge the support from the $\mathrm{PhD}$ research startup foundation of Guangdong Medical University (2XB14006).

\section{References}

1. M. A. Yücel, J. Selb, R. J. Cooper, D. A. Boas, "Targeted principle component analysis: A new motion artifact correction approach for near-infrared spectroscopy," J. Innov. Opt. Health Sci. 7, 342-345 (2014).

2. T. Li, Y. Li, Y. Sun, M. Duan, L. Peng, "Effect of head model on Monte Carlo modeling of spatial sensitivity distribution for functional near-infrared spectroscopy," J. Innov. Opt. Health Sci. 8, 1550024 (2015).

3. S. Brigadoi, L. Ceccherini, S. Cutini, F. Scarpa, P. Scatturin, J. Selb, L. Gagnon, D. A. Boas, R. J. Cooper, "Motion artifacts in functional near-infrared spectroscopy: A comparison of motion correction techniques applied to real cognitive data," Neuroimage 85, 181-191 (2014).

4. L. Zhang, J. Sun, B. Sun, C. Gao, H. Gong, "detecting bilateral functional connectivity in the prefrontal cortex during a Stroop task by near-infrared spectroscopy," J. Innov. Opt. Health Sci. 6, 1350031 (2013).

5. J. Sun, B. Sun, L. Zhang, Q. Luo, H. Gong, "Correlation between hemodynamic and electrophysiological signals dissociates neural correlates of conflict detection and resolution in a Stroop task: A simultaneous near-infrared spectroscopy and eventrelated potential study," J. Biomed. Opt. 18, 096014-096014 (2013).

6. C. Gao, J. Sun, X. Yang, H. Gong, "Gender differences in brain networks during verbal Sternberg tasks: A simultaneous near-infrared spectroscopy and electro-encephalography study," J. Biophoton. (2017).

7. Z. Zhang, M. Schneider, U. Fritschi, I. Lehner, R. Khatami, "Near-infrared spectroscopy (NIRS) as a useful tool to evaluate the treatment efficacy of positive airways pressure therapy in patients with obstructive sleep apnea syndrome (OSAS): A pilot study," J. Innov. Opt. Health Sci. 7, 342-345 (2014).

8. M. Ferrari, V. Quaresima, "A brief review on the history of human functional near-infrared spectroscopy (fNIRS) development and fields of application," Neuroimage 63, 921-935 (2012).

9. X. Cui, D. M. Bryant, A. L. Reiss, "NIRS-based hyperscanning reveals increased interpersonal coherence in superior frontal cortex during cooperation," Neuroimage 59, 2430-2437 (2012).

10. T. J. Huppert, S. G. Diamond, M. A. Franceschini, D. A. Boas, "HomER: A review of time-series analysis methods for near-infrared spectroscopy of the brain," Appl. Opt. 48, D280-D298 (2009).

11. I. Tachtsidis, T. S. Leung, L. Devoto, D. T. Delpy, C. E. Elwell, "Measurement of Frontal Lobe Functional Activation and Related Systemic Effects: A Near-Infrared Spectroscopy Investigation," Adv. Exp. Med. Biol. 614, 397-403 (2008).

12. S. Patel, T. Katura, A. Maki, I. Tachtsidis, "Quantification of systemic interference in optical topography data during frontal lobe and motor cortex activation: An independent component analysis," Adv. Exp. Med. Biol. 701, 45-51 (2011).

13. I. Tachtsidis, A. Papaioannou, "Investigation of frontal lobe activation with fNIRS and systemic changes during video gaming," Adv. Exp. Med. Biol. 789, 89-95 (2013).

14. G. Durantin, J. F. Gagnon, S. Tremblay, F. Dehais, "Using near infrared spectroscopy and heart rate variability to detect mental overload," Behav. Brain Res. 259, 16 (2014).

15. M. Tanida, M. Katsuyama, "Relation between mental stress-induced prefrontal cortex activity and skin conditions: A near-infrared spectroscopy study," Brain Res. 1184, 210-216 (2008).

16. B. Sun, L. Zhang, H. Gong, J. Sun, Q. Luo, "Detection of optical neuronal signals in the visual cortex using continuous wave near-infrared spectroscopy," Neuroimage 87, 190-198 (2014).

17. A. V. Medvedev, J. M. Kainerstorfer, S. V. Borisov, A. H. Gandjbakhche, J. VanMeter, "Seeing" 
electroencephalogram through the skull: imaging prefrontal cortex with fast optical signal," $J$. Biomed. Opt. 15, 061702 (2010).

18. G. Gratton, M. Fabiani, "Fast optical imaging of human brain function," Front. Hum. Neurosci. 4, 52 (2010).

19. G. Gratton, P. M. Corballis, "Removing the heart from the brain: Compensation for the pulse artifact in the photon migration signal," Psychophysiology 32, 292-299 (1995).

20. Z. Zhang, B. Sun, H. Gong, L. Zhang, J. Sun, B. Wang, Q. Luo, "A fast neuronal signal-sensitive continuous-wave near-infrared imaging system," Rev. Sci. Instrum. 83, 094301 (2012).
21. Z. Zhao, L. Liu, C. Zhang, H. Li, J. Wang, "Research and analysis of morphological filter's structure element selection principle," Power System Protection and Control 37, 21-25 (2009).

22. T. Li, M. Yang, X. Zhou, S. Cheng, "Method of partial discharge signal analysis based on wavelet transform and mathematical morphology," Power Syst. Technol. 6, 5 (2007).

23. W. D. Chen, L. I. Xin, J. Liu, Y. Y. Hao, Y. X. Liao, S. U. Yu, S. M. Zhang, X. X. Zheng, "Mathematical morphology based electro-oculography recognition algorithm for human-computer interaction," $J$. Zhejiang Univ. 45, 644-649 (2011). 